Article

\title{
Effect of Thickness Ratio Coefficient on the Mixture Transportation Characteristics of Helical-Axial Multiphase Pumps
}

\author{
Wei Han ${ }^{1,2, *}$, Xing $\mathrm{Li}^{1}$, Youliang $\mathrm{Su}^{1}$, Min Su ${ }^{3, *}$, Rennian $\mathrm{Li}^{1,2}$ and $\mathrm{Yu}$ Zhao ${ }^{1}$ \\ 1 Department of Energy and Power Engineering, Lanzhou University of Technology, Lanzhou 730050, China; \\ Lut205L@163.com (X.L.); liangle88@126.com (Y.S.); lirn@lut.cn (R.L.); z2856138060@163.com (Y.Z.) \\ 2 Key Laboratory of Fluid Machinery and Systems, Lanzhou University of Technology, Lanzhou 730050, China \\ 3 Department of Electrical and Information Engineering, Lanzhou University of Technology, \\ Lanzhou 730050, China \\ * Correspondence: hanwei@lut.cn (W.H.); sumin@lut.cn (M.S.)
}

Received: 13 November 2019; Accepted: 31 December 2019; Published: 2 January 2020

\begin{abstract}
With the decrease of oil and gas resources on land, increased attention has been paid to multiphase oil-gas exploitation and the transportation technology represented by oil-gas multiphase pumps. The helical-axial multiphase pump has become the focus of research on oil and gas mixed transmission technology due to its relatively high operating efficiency and adaptability to a wide range of gas volume fraction changes. In order to investigate the thickness variation in the air foil from the hub to the shroud of the blade on the mixture transportation characteristics of the gas-liquid two-phase flow in a helical-axial pump, the thickness ratio coefficient $\xi$ was introduced, and the hydraulic performance of the single compression unit with different thickness ratio coefficients was investigated. A single compression unit including an impeller, diffuser, inlet section and outlet section of a helical-axial multiphase pump. The hydraulic performance including the hydraulic head and efficiency was investigated by numerical simulation with the Eulerian multiphase model and the shear stress transport (SST) $k-w$ turbulence model. In order to demonstrate the validity of the numerical simulation approach, the hydraulic head and efficiency of the basic model was measured based on a gas-liquid two-phase flow pump performance test bench. The simulation results agreed well with the experimental results; the error between the simulation results and experimental results of different inlet gas volume fractions was within $10 \%$ at the design point, which indicated the numerical simulation method can be used in the research. The thickness ratio coefficient $\xi$, which was taken as a variable, and the aggregation degree $\lambda$ of the gas were introduced to analyze the gas-liquid mixture transportation characteristics of the pump. The thickness ratio coefficient was selected in a range from 0.8 to 1.8. The results showed that, for the same hub thickness, the head coefficient and efficiency increase, and the aggregation degree of gas decreases with the decreasing of the thickness ratio coefficient. The head coefficient of the modification multiphase pump was $5.8 \%$ higher in comparison to the base pump while the efficiency was 3.1\% higher than that of the base pump, the aggregation degree of this model was the lowest, which was $30.3 \%$; the optimal model in the research was the model of scheme 1 with $\xi=0.8$. The accumulation of gas in the flow passage of the impeller could be delayed to the trailing edge of the blade by adjusting the thickness ratio coefficient, which produced a super-separated airfoil for helical-axial multiphase pumps and effectively ensured reliable operation under high gas volume fraction conditions. The accumulation area of gas was consistent with the area in which the gradient of turbulent kinetic energy was large.
\end{abstract}

Keywords: helical-axial multiphase pump; mixture transportation characteristics; thickness ratio coefficient; aggregation degree; super-separated airfoil 


\section{Introduction}

With the continuous development of society, oil field exploitation and transportation technology requirements in industrial applications are continually increasing. The conventional conveying equipment, which includes liquid-phase pumps, compressors and separators, is being replaced by multiphase pumps. Multiphase pumps directly transport the mixture of oil, gas and water from subsea oil wells through a single pipeline instead of two pipelines, which can simplify equipment usage and save capital costs [1]. As the core of oil-gas mixture transportation technology, the design and application of multiphase pumps has become a research hotspot. At present, two main types of multiphase pumps are widely used in the energy industry. The first is the twin-screw multiphase pump; the advantage of this is that the gas-liquid two-phase medium is more stable and the change in gas volume fraction has little effect on the performance of the pump. The disadvantage is that the conveying flow is low, and it is sensitive to particles such as sediment. This type of pump has low reliability, short lifespan and its maintenance is inconvenient [2]. The other pump type is the helical-axial-flow oil-gas multiphase pump; the advantage of this kind of pump is that the structure of the impeller has a long, square channel with a larger flow curvature radius and the ability for large conveying flow, with a higher gas-liquid two-phase transportation capacity than other centrifugal pumps and mixed-flow pumps. When the centrifugal pump conveys the gas-liquid two-phase medium with a certain inlet gas volume fraction, the efficiency will drop sharply and it becomes difficult even to maintain the operation of the pump [3]. The helical-axial multiphase pump can transport a gas-liquid multiphase flow with a gas volume fraction from $0 \%$ to $90 \%$; it can continue to work under the condition of high inlet gas volume fraction [4]. The disadvantage is that the performance of the pump will decrease with the increase in the gas volume fraction [5]. Combined with the advantages, the application of multiphase pumps is an ideal choice for gas-liquid two-phase transportation in the petroleum industry. The characteristics of different types of multiphase pumps are listed in Table 1.

Table 1. Characteristics of different types of multiphase pumps.

\begin{tabular}{ccc}
\hline Type of Pump & Advantage & Disadvantage \\
\hline $\begin{array}{c}\text { Twin-screw } \\
\text { multiphase pump }\end{array}$ & $\begin{array}{c}\text { Stable operation; the performance is not } \\
\text { sensitive to gas volume fraction }\end{array}$ & Short lifespan; inconvenient maintenance \\
\hline $\begin{array}{c}\text { Helical-axial } \\
\text { multiphase pump }\end{array}$ & $\begin{array}{c}\text { Large conveying flow; higher gas-liquid } \\
\text { two-phase transportation capacity with the } \\
\text { high inlet gas volume fraction; }\end{array}$ & $\begin{array}{c}\text { Performance degrades with the increasing of inlet } \\
\text { gas volume fraction }\end{array}$ \\
\hline Centrifugal pump & Simple structure and easy to manufacture & $\begin{array}{c}\text { Low gas volume fraction; the efficiency will drop } \\
\text { sharply when the gas volume fraction is above 10\% }\end{array}$ \\
\hline
\end{tabular}

The initial application of the multiphase pump was conducted in the 1990s: the world's first multiphase pump, the Poseidon helical-axial multiphase pump developed by the French Petroleum Institute (IFP), Norwegian National Oil Company (Statoil) and France's Total Oil Company was installed by Shell, which successfully boosted the well effluent from a satellite well to the host platform six miles away [6]. Subsequently, various investigations of multiphase pumps have been carried out. To date, hundreds of multiphase pumps have been used all over the world, including in desert oil fields [7], marginal oil fields [8] and deep-sea oil fields [9,10]. The helical-axial multiphase pumps are widely used at present, but the gas-liquid two-phase flow is easily affected by many external factors, and relative motions occur between phases and complex interface effects, creating problems for determining the evolution mechanism of two-phase flow [11,12]. Therefore, the significant issues for improving the performance of multiphase pumps are the design and optimization of multiphase pumps since they are directly related to the pump performance [13].

Many scholars have carried out research into the multiphase pumps since the 1990s, and they have contributed a great deal to the design and internal flow analysis of multiphase pumps. Ma et al. [14] designed an impeller by combining axial pump and axial compressor theory and built a three-dimensional (3D) model. Wang et al. [15] simulated the two-phase 3D turbulence flow characteristics numerically 
under different conditions based on the Euler multi-fluid model method and discussed the internal flow characteristics of the helical multiphase pump-the hydraulic performance can be investigated by giving different inlet gas volume fractions as the boundary condition-and the results showed that the model can accurately predict the performance of the multiphase pump. Yu et al. [16] used numerical simulation to analyze gas-liquid two phase flow; the numerical results demonstrated that gas blocking could be effectively avoided if a relatively small radical dimension is adopted in the flow passage. Li et al. [17] explored the impeller design method and analyzed the mechanism of the inner flow, and finally proposed a design method for the incompressible medium; they introduced the design and experimental study of the principle machine, and the results showed that the pressurization performance of a multiphase pump can be improved by reducing the inlet impact angle and increasing the outlet angle. Cao et al. [18] combined the inverse method and direct flow analysis methods for the hydrodynamic design of a gas-liquid two-phase flow rotodynamic pump impeller; the impeller blade was back-loaded at the bub side to produce a favorable pressure distribution and the results demonstrated that the designed pump worked well and the design computation validity was proven. China Petroleum University has conducted hydraulic and structural designs, flow mechanism analysis, performance prediction and other work on helical-axial multiphase pumps and completed the performance test research on three generations of prototypes [19-21], providing important references for scholars in terms of blade selection, head estimation and performance prediction for this pump in the future.

There are also increasing numbers of researchers focusing on the improvement of multiphase pump performance. In research on the performance improvement of multiphase pumps, one of the most successful is the helical-axial multiphase pump developed by Korean Hanyang University (Seoul, South Korea), which uses Latin hypercube technology [22,23] to optimize variables by sampling the best parameters from multiple variables to optimize the parameters to achieve high pump efficiency. $\mathrm{Li}$ et al. [24] used the orthogonal experimental method to optimize a multiphase pump to improve its efficiency; the optimization result showed that the relative head and efficiency increased. Yi Shi et al. [25] investigated the two-phase flow characteristics in a normal impeller and split vane impeller at different inlet gas volume fractions (GVFs), and the simulation results demonstrated that the modified impeller with split vanes can effectively suppress gas accumulation in the flow passage and improve the flow conditions compared to that of the normal impeller. The results of the research will be a base for further study on measures to diminish gas pockets in a flow passage with a high inlet GVF. Him-Chan Lee et al. [26] presented a procedure for the design optimization of a multiphase pump, with the evaluation of influences performed by using the $2^{\mathrm{K}}$ factorial method and the efficiency of multiphase pump derived by using the response surface approximation (RSA) model; the results showed the method was effective for improving the performance of multiphase pumps, which suggests guidelines for the high-performance design of a multiphase pump through numerical optimization techniques. Shi et al. $[27,28]$ analyzed the pressurization performance of the multiphase impeller under different conditions and studied the load distribution characteristics of the impeller blades. They showed that the pressurization performance of the impeller is stronger in the first half of the impeller when close to the rim, and the main part of the energy conversion occurs in the first one-third of the impeller. The results provided an important theoretical basis for the optimization of impeller design.

The helical-axial multiphase pump is a multiphase conveying device that integrates the performance of the conventional liquid phase pump and a gas compressor. Multiphase pumps differ from single-phase pumps in performance evaluation, internal flow field and design techniques. Due to the complicated interphase interactions between gas and liquid, including flow separation and gas pockets, the transporting efficiency of multiphase pumps is unsatisfactory and requires further research [29]. The gas blocking phenomenon affected the operation of the multiphase pump seriously, so enhancing the mixing of the two-phase gas-liquid will be one of the principal tasks in future research. Combined with the visual observation of the internal flow, the performance of the pump will be greatly improved. Some related studies about gas-liquid mixing and visual analysis techniques have already been carried out. Farooq Sher et al. [30] developed an innovative approach to address the 
behavior of gas-liquid mixing by using electrical resistance tomography, and the technique provided clear visual evidence of better mixing that took place inside the gas-liquid system. Scholars can refer to this technology to improve the accuracy of the observation of the internal flow field in the multiphase pump, thus providing evidence for improving the performance of the multiphase pump.

The impeller, as the core component of the multiphase pump, considerably influences the performance of the pump, so it is particularly important to improve the performance of the multiphase pump by improving the performance of the impeller. The thickness of the blade is an important parameter that affects the performance of pumps, but the research is lacking on the impeller blade thickness coefficient of the multiphase pump in the current study. The thickening law of the blade has a great influence on the performance of impeller, thus affecting the performance of the multiphase pump. Therefore, taking the study of gas-liquid multiphase pumps as our starting point, we analyzed the internal flow characteristics of the impeller by changing the thickness ratio coefficient $\xi$ in this study, comparing the aggregation degree $\lambda$ of the gas phase of different schemes and obtaining a super separation airfoil that can delay the separation of gas and liquid to the trailing edge as much as possible, thereby providing a reference method for the modification design of the impeller and the improvement of the mixture transportation capacity of gas-liquid multiphase pumps.

\section{Design and Modeling of Helical-Axial Multiphase Pump}

\subsection{Parameter Determination}

According to the requirements of the research and development program projects, the design parameters are listed in Table 2.

Table 2. Design parameters.

\begin{tabular}{cccc}
\hline Design Flow Rate $\left(\mathrm{Q}_{0}, \mathbf{m}^{\mathbf{3}} \mathbf{h}^{-\mathbf{1}}\right)$ & Hydraulic Head $(\mathrm{H}, \mathrm{m})$ & Rotational Speed $(\mathrm{n}, \mathrm{rpm})$ & Efficiency $(\eta, \%)$ \\
\hline 100 & 30 & 4500 & 62 \\
\hline
\end{tabular}

\subsection{Model Establishment}

Considering the complexity of the model and the large number of grids leading to a longer numerical calculation, we selected a single compression unit of the multiphase pump for numerical simulation, which included the impeller, diffuser, inlet section and outlet section. The main parameters of the impeller and diffuser are shown in Table 3. We built a 3D geometric model of the compression unit including the impeller and diffuser with the 3D drawing software SolidWorks (education column) based on the hydraulic design, as shown in Figure 1.

Table 3. Main parameters of the hydraulic parts of a multiphase pump.

\begin{tabular}{ccc}
\hline Hydraulic Parts & Main Structural Parameter & Value \\
\hline \multirow{4}{*}{ Impeller } & Diameter $D(\mathrm{~mm})$ & 150 \\
& Number of blades $Z$ & 4 \\
& Half cone angle of the hub $\gamma\left({ }^{\circ}\right)$ & 6 \\
Axial length $e(\mathrm{~mm})$ & 55 \\
& Inlet angle of the blade $\beta_{1}$ & 10 \\
Outlet angle of the blade $\beta_{2}$ & 14 \\
\hline Diameter $D_{d}(\mathrm{~m})$ & 150 \\
Diffuser & Number of blades $Z_{d}$ & 17 \\
& Half cone angle of the hub $\gamma_{\mathrm{d}}\left(^{\circ}\right)$ & 6 \\
& Axial length $e_{d}($ mm) & 55 \\
& Inlet angle of the blade $\beta_{d 1}$ & 38 \\
& Outlet angle of the blade $\beta_{d 2}$ & 90 \\
\hline
\end{tabular}






Figure 1. The model of the impeller and diffuser.

\subsection{Mesh Generation}

The mesh generator ICEM-CFD (education column) is used in the research, which is a comprehensive mesh generation tool. It is one of the most widely used mesh generator in recent years. It can also complete the mesh generation of models with complex geometric structure, because this mesh generator has the features such as model repair and mesh editing, so the high-quality mesh can be obtained. The impeller of the helical axial multiphase pump has complex structure with square channel and large curvature radius, so the mesh generator ICEM-CFD is employed to divide the unstructured mesh of the computational domain. The unstructured grid, with more flexibility and adaptability to the complex model, was used to divide the hydraulic parts of the multiphase pump. This kind of grids has no regular topological structure and no concept of layers. The distribution of grid nodes is arbitrary, so it has the property of flexibility and the distribution of the divided grids is reasonable. The rationality of grid distribution also affects the accuracy of numerical simulation, the unstructured grids has the reasonable distribution of grids, in addition, the grids near the blade are locally encrypted because the flow condition of this area changes dramatically. In order to ensure the accuracy of the numerical calculation and save the calculation time, the denser grids are distributed in the area where the flow field changes greatly, so the flow condition near this area can be described in more detail. The structured grid was used to divide the inlet and outlet sections using a simple structure. The overall grids were of high-quality at above 0.35 , so the grids can be used in numerical simulations. The value 0.35 represents the quality of the grids. The criterion of grids quality generally includes orthogonality and smoothness of the grids [31]. Orthogonality of the grids means that the lines or faces of the grids should be as orthogonal as possible, the better the orthogonality, the higher the grids quality. The smoothness of the grids is usually represented by the dimensional change ratio between adjacent grids. The dimensional change ratio between adjacent grids should not exceed 2 , which means the transition of grids is gradual, not a sudden change. The mesh smoothing function can be used if the smoothness of the grids is poor. There are certain requirements for the grids that can be used in numerical simulation calculations, such as the grids need to be checked and the quality of the grids needs to be higher than a certain value. The unstructured grids will be checked for errors after dividing, such as if there are duplicate element or missing internal faces. It must be guaranteed that there are no errors of grids, so the grids will meet the requirement for numerical simulation. According to the professional literature [32], the minimum quality of the grids used for numerical simulation is at least 0.2 . The quality of grids can affect the accuracy of numerical simulation. If the quality of grids is less than 0.2 , the accuracy of the numerical simulation will be reduced. The quality of grids in the manuscript has been checked and is higher than 0.35 , which is above the minimum requirement for grids quality, the grids can be used in numerical simulations.

To reduce the influence of the grid density on the numerical simulation results, all five sets of mesh are employed to conduct the independence test of mesh number under the design condition with an inlet gas volume fraction of $30 \%$. Table 4 shows the predicted results of the hydraulic head and efficiency of the multiphase pump with different mesh schemes; it can be seen that the differences 
of the hydraulic head and efficiency in schemes 3,4 and 5 were relatively small. Therefore, the total number of cells was finally selected as 7.541 million for numerical simulation to reduce the required computing resources. The overall grids of the single compression unit and an enlarged local grid drawing in the black frame of the top figure are depicted in Figure 2.

Table 4. Independence test of mesh number.

\begin{tabular}{cccccc}
\hline \multicolumn{7}{c}{ Mesh Scheme } \\
\hline Components & $\mathbf{1}$ & $\mathbf{2}$ & $\mathbf{3}$ & $\mathbf{4}$ & $\mathbf{5}$ \\
\hline Inlet domain & $1,522,500$ & $1,522,500$ & $1,522,500$ & $1,522,500$ & $1,522,500$ \\
Impeller & 625,907 & $1,193,245$ & $2,761,281$ & $3,618,690$ & $4,780,106$ \\
Diffuser & 772,633 & $1,417,004$ & $1,735,270$ & $2,458,466$ & $3,177,553$ \\
Outlet domain & $1,522,500$ & $1,522,500$ & $1,522,500$ & $1,522,500$ & $1,522,500$ \\
Total meshes & $4,443,540$ & $5,655,249$ & $7,541,551$ & $9,122,156$ & $11,002,659$ \\
Hydraulic head & 32.61 & 31.86 & 31.4 & 31.36 & 31.33 \\
Efficiency & $68.1 \%$ & $67.3 \%$ & $66.2 \%$ & $66.17 \%$ & $66.13 \%$ \\
\hline
\end{tabular}

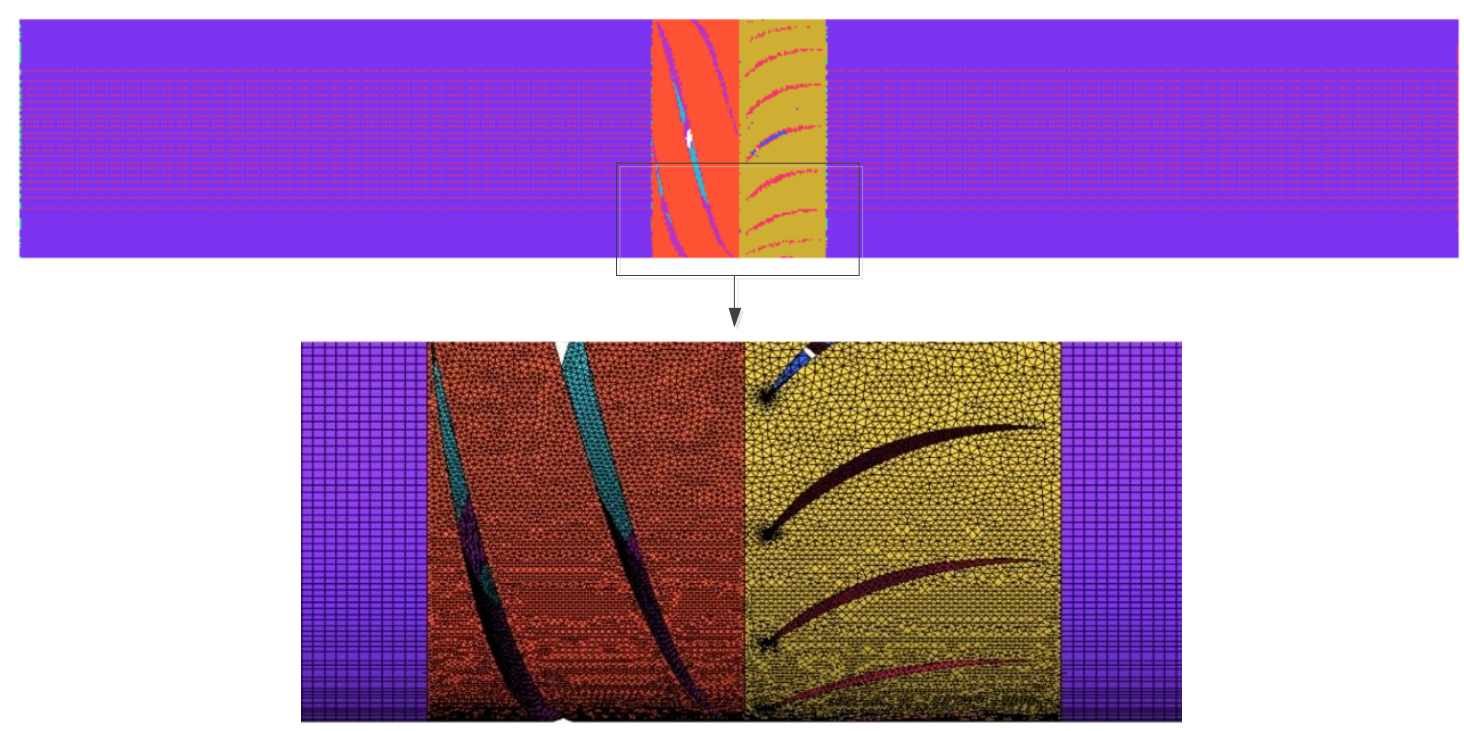

Figure 2. Grids of a single compression unit.

\section{Numerical Simulation and Results Analysis}

\subsection{Numerical Method}

With the pressure increase of the multiphase pump, the gas volume fraction changes with the work process. It is assumed that the gas-liquid two-phase flow is incompressible bubble flow. The compressibility of gas can be determined by the Mach number. The compressibility of gas is not considered when the Mach number is less than 0.3. Since the speed of the fluid in the helical-axial multiphase pump is far less than the local speed of sound, its Mach number is less than 0.3. Therefore, the compressibility of the gas phase is not considered in the numerical simulation of the article [33]. Considering the high-volume fraction of the gas phase and the stability and rationality of the simulation results, we selected the high-accuracy Eulerian multiphase model to conduct the steady-state calculation in the numerical simulation. The hydraulic performance of the multiphase pump with different thickness ratio coefficient schemes can be investigated by the numerical simulation method, the distribution of gas-phase can also be obtained by the steady-state simulation. The steady-state simulation has the characteristics of reliable results and easy convergence; therefore, the steady-state simulation is adopted in the research. The Eulerian multiphase model is suitable for mixed or separated flows. It has a good effect in simulating the aggregation and fragmentation of gas and is commonly used. The Eulerian multiphase 
model establishes a momentum equation and continuity equation for each phase; the interaction between the two phases and the transfer of physical quantities on the phase interface are considered in the model, so the flow characteristics of the research object can be described more accurately [34]. The shear stress transport (SST) $k$ - $w$ turbulence model, which is appropriate for the prediction of flow separation among two-equation linear eddy-viscosity models, was adopted [35]. This model combines the advantage of $k-w$ model in simulating the near-wall flow and that of the $k-\varepsilon$ model in simulating flow outside the boundary layer [36]. Thus, the SST $k-w$ turbulence model can be used to obtain a very high accuracy and stability of the solution. In addition, it has been found that SST $k-w$ turbulence model does a better job of predicting flow field in impeller than other turbulence models [37]; it is widely used in relevant studies on gas-liquid two-phase flow $[38,39]$. The pressure-based solver can provide Eulerian multiphase model, which is adopted in the numerical simulation. The pressure-based solver is mainly used in the incompressible flow and has the feature of fast convergence, the gas-liquid two-phase flow in the research is incompressible, so the pressure-based solver is employed in the research. The phase-coupled SIMPLE scheme was used in the pressure-velocity coupling calculation.

The applicability of different multiphase models and turbulence models [40] are shown in Tables 5 and 6.

Table 5. The applicability of different multiphase models.

\begin{tabular}{cl}
\hline Multiphase Models & \multicolumn{1}{c}{ Applicability } \\
\hline Mixture & $\begin{array}{l}\text { A simplified multiphase model, which is suitable for gas-liquid two-phase flow } \\
\text { with low gas phase fraction. There is only one set of momentum equations, and the } \\
\text { continuity equation can be solved for gas-liquid two-phase flow. }\end{array}$ \\
\hline Eulerian & $\begin{array}{l}\text { The model establishes the momentum equation and continuity equation for each } \\
\text { phase, which can describe the two-phase flow well and has a high accuracy of } \\
\text { calculation. }\end{array}$ \\
\hline VOF & $\begin{array}{l}\text { The model is suitable for two-phase flow with obvious stratification and is used to } \\
\text { deal with the problem of multiphase flow without interpenetration. }\end{array}$ \\
\hline
\end{tabular}

Table 6. The applicability of different turbulence models. SST: shear stress transport.

\begin{tabular}{cl}
\hline Turbulence Models & \multicolumn{1}{c}{ Applicability } \\
\hline Standard $k-\varepsilon$ model & $\begin{array}{l}\text { A simplified multiphase model, which is suitable for gas-liquid two-phase flow } \\
\text { with low gas phase fraction. There is only one set of momentum equations, and the } \\
\text { continuity equation can be solved for gas-liquid two-phase flow. }\end{array}$ \\
\hline \multirow{2}{*}{ RNG $k-\varepsilon$ model } & $\begin{array}{l}\text { The model can deal with the turbulent flow problem with a low Reynolds number. } \\
\text { The influence of the vortex in the turbulent flow is considered in the RNG } k-\varepsilon \\
\text { model, it has higher accuracy than the standard } k-e \text { model. }\end{array}$ \\
\hline SST $k-w$ & $\begin{array}{l}\text { The model considers the effect of shear stress on turbulent flow, can deal with free } \\
\text { flow in the near-wall region and is often used for flows with large eddies. } \\
\text { It has high accuracy and reliability. }\end{array}$ \\
\hline
\end{tabular}

Combined with the application of different multiphase models and turbulence models, the Eulerian multiphase model and SST $k-w$ are the most suitable models that can be used to the numerical simulation of the gas-liquid two-phase flow in the research.

\subsection{Boundary Condition}

The inlet boundary condition was the velocity-inlet, assuming that the inlet gas-liquid two-phase was uniformly mixed. The primary phase was set as water and the secondary phase as air. The secondary phase volume fraction was the inlet gas volume fraction. The outlet boundary condition is defined as the pressure outlet, and the reference pressure is the default pressure. In the numerical simulation, the no-slip boundary condition was applied to the flow boundary, because the velocity of fluid on 
the surface of wall is zero. The no-slip boundary condition is generally adopted in the viscous flow and the analysis of large-scale flow [41]. For example, if the scale is less the $10^{-6}$ order of magnitude, the slip boundary condition can be adopted in the flow. The standard wall function method was applied to complement the turbulence model near the boundary. The physical quantities on the wall are connected with those in the turbulent core, it has the advantages of less computation and better accuracy [42], so the flow field near the wall can be calculated.

\subsection{Setting of the Numerical Calculation}

The rotating coordinate system was adopted for the impeller region and a stationary coordinate system was adopted for the diffuser, inlet, and outlet region. We used a dynamic-static interface for data transmission between the dynamic and static surfaces. The residual of convergence was set to 0.00001. In the progress of numerical calculation, the monitoring window was opened to observe the residual variation of the variables, the pressure of the inlet and outlet, and the torque. The calculation ended when the calculated residual was lower than the residual set before calculation, or the monitoring value was stable and did not change with the calculated step size of 10,000 steps.

\subsection{Governing Equations}

The equations governing gas-liquid two-phase flow are provided in Equations (1) to (5):

The continuity equation is

$$
\nabla \cdot\left(\alpha_{k} \rho_{k} U_{k}\right)=0
$$

The momentum equation is

$$
\nabla \cdot\left(\alpha_{k} \rho_{k} U_{k} U_{k}-\alpha_{k} \tau_{k}\right)=-\alpha_{k} \nabla p+M_{k}+\alpha_{k} \rho_{k} f_{k}
$$

where $k$ means the $\mathrm{k}$ phase: if $k=\mathrm{g}$, means gas, if $k=1$, means liquid. $\alpha_{k}$ is the volume fraction of the phase. $\rho_{k}$ denotes the density of the phase, and its unit is $\mathrm{kg} / \mathrm{m}^{3} . p$ represents the pressure, and its unit is pa. $U_{k}$ is the velocity of the phase, and its unit is $\mathrm{m} / \mathrm{s} . M_{k}$ is the interaction force between phases and the unit is $\mathrm{N} \cdot \mathrm{m} . f_{k}$ denotes the mass force, and its unit is $\mathrm{N} \cdot \mathrm{m}$.

where $\tau$ denotes the viscous stress tensor concerning fluid viscosity as well as turbulence viscosity. Its components are written in the following form:

$$
\tau_{k, i j}=2 \mu_{k} s_{i j}-\overline{\rho_{k} w_{k i}{ }^{\prime} w_{k j}{ }^{\prime}}
$$

where $i, j=1,2,3$ represent the three directions of a relative Cartesian coordinate system $\left(x_{1}, x_{2}, x_{3}\right)$ rotating with the impeller. $s_{i j}$ denotes the strain tensor. $-\overline{\rho_{k} w_{k i}{ }^{\prime} w_{k j}{ }^{\prime}}$ represent the turbulent Reynolds stresses due to turbulent motion. The turbulent Reynolds stress tensor is given in following form:

$$
-\overline{\rho_{k} w_{k i}{ }^{\prime} w_{k j}{ }^{\prime}}=2 \mu_{t} s_{i j}-\frac{2}{3} \rho_{k} k \delta_{i j}
$$

where the solution of $\mu_{t}$ is based on shear stress transport (SST) $k-w$ turbulence model. The formula of $\mu_{t}$ is given as follows:

$$
\mu_{t}=\frac{\rho_{m} a_{1} k}{\max \left(a_{1} \omega, S F_{2}\right)}
$$

where $\mu_{t}$ is the eddy viscosity-the unit is pa.s- $\mathrm{a}_{1}=5 / 9, \mathrm{k}$ is turbulent kinetic energy, $\omega$ is turbulent frequency, $S$ is the invariant of the strain rate and $F_{2}$ is a blending function, which restricts the limiter to the wall boundary layer [43]. 
The volume fraction of each phase when performing the numerical simulation is determined according to the following equation:

$$
\begin{aligned}
& \alpha_{l}=\frac{Q_{l}}{Q_{l}+Q_{g}} \times 100 \%, \\
& \alpha_{g}=\frac{Q_{g}}{Q_{l}+Q_{g}} \times 100 \%,
\end{aligned}
$$

The relationship of the volume fraction between the gas and liquid is shown in Equation (8):

$$
\alpha_{l}+\alpha_{\mathrm{g}}=1,
$$

where $Q_{l}$ denotes the flow rate of the liquid phase while $Q_{g}$ denotes the flow rate of the gas phase; the units are $\mathrm{m}^{3} / \mathrm{h}$.

The mixture density of the gas-liquid two-phase is defined as

$$
\rho_{m}=\alpha_{\mathrm{g}} \rho_{g}+\alpha_{l} \rho_{l}
$$

The hydraulic performance of the multiphase pump including the hydraulic head and efficiency is defined as follows:

$$
H=\frac{p_{2}-p_{1}}{\rho_{m} g}
$$

where $p_{1}, p_{2}$ denote the inlet and outlet pressure of the multiphase pump, respectively.

The efficiency of the multiphase pump can be calculated by the following equation:

$$
\eta=\frac{\rho_{m} g Q H}{M \omega}
$$

where $M$ represents the torque of multiphase pump and $\omega$ is the angular velocity of the impeller.

\subsection{Analysis of Simulation and Experimental Results}

Figure 3 shows the schematic diagram and the picture of the gas-liquid two-phase flow pump performance test bench. Experiments were performed with a mixture of air and water. During the progress of the experiment, the speed of the pump was regulated by an electromotor; an air compressor was used to pressurize the gas. The flow rate of liquid was regulated by the liquid inlet valve and the electromagnetic flowmeter, and the gas flow rate into the pipeline was regulated by the air flowmeter and air inlet valve. Pressure gauges for the inlet and outlet were used to measure the differential pressure to calculate the hydraulic head of the multiphase pump. The torque was tested using a torque sensor to calculate the efficiency of the multiphase pump.

The hydraulic head and efficiency of the multiphase pump under the condition of $0.6,0.8,1,1.2$, and 1.4 times the designed flow rate $Q_{0}$ with the inlet gas volume fractions of $30 \%$ and $50 \%$ were predicted with the numerical simulation, respectively, as shown in Figure 4. The experimental results of different flow rates with inlet gas volume fractions of $30 \%$ and $50 \%$ are also shown in Figure 4 . It can be seen that the simulation results were in good agreement with the experimental results. The numerical simulation hydraulic head $(H)$ and efficiency $(\eta)$ of the single compression unit of the inlet gas volume fraction of $30 \%$ were $31.4 \mathrm{~m}$ and $66.2 \%$ under the designed conditions, respectively. The experimental head was $29.2 \mathrm{~m}$ while the efficiency was $60 \%$ under the designed condition. It can also be seen from Figure 4 that the numerical simulation hydraulic head $(H)$ and efficiency $(\eta)$ of the single compression unit of the inlet gas volume fraction of $50 \%$ were $29.32 \mathrm{~m}$ and $65.96 \%$ under the designed conditions while the corresponding experimental values were $27.12 \mathrm{~m}$ and $57.89 \%$, respectively. The error between the simulation results and experimental results of different inlet gas volume fractions was within $10 \%$ at the design point, which was reasonable because the gas-liquid two-phase flow had a certain loss in the 
actual progress of operation. The external characteristics can indirectly verify the accuracy of numerical simulation, and the internal flow characteristics can be inferred based on external characteristics. This proved that the method used in the numerical simulation is reliable, and indicated that we can use this method for the modification design of the impeller in the subsequent study. As the flow rate increased, the numerical and experimental hydraulic head decreased continually. The efficiency reached a maximum at $1.2 Q_{0}$, and the range of high efficiency was wide.

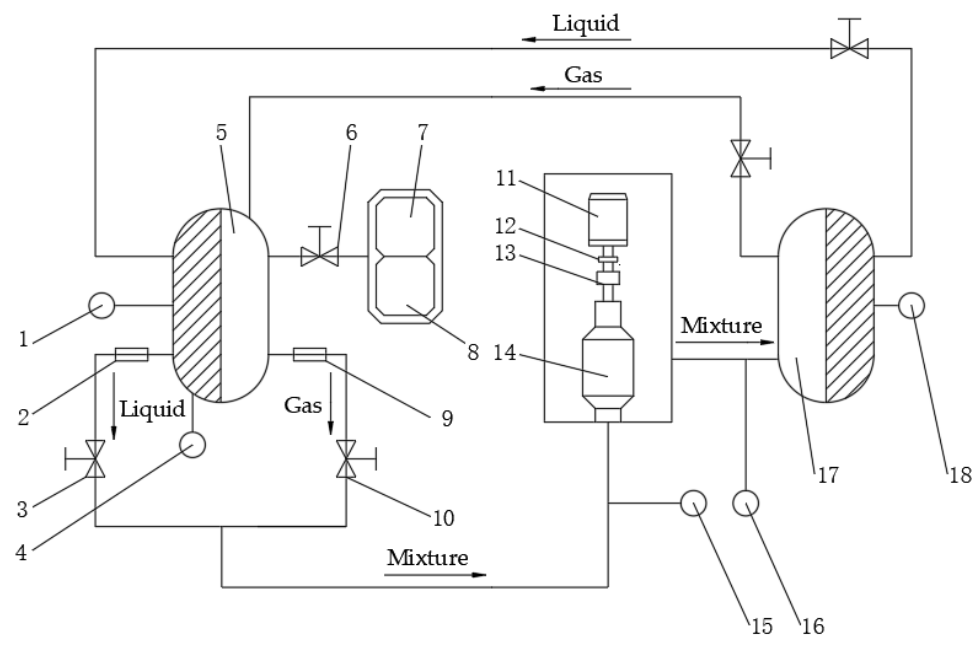

(a)

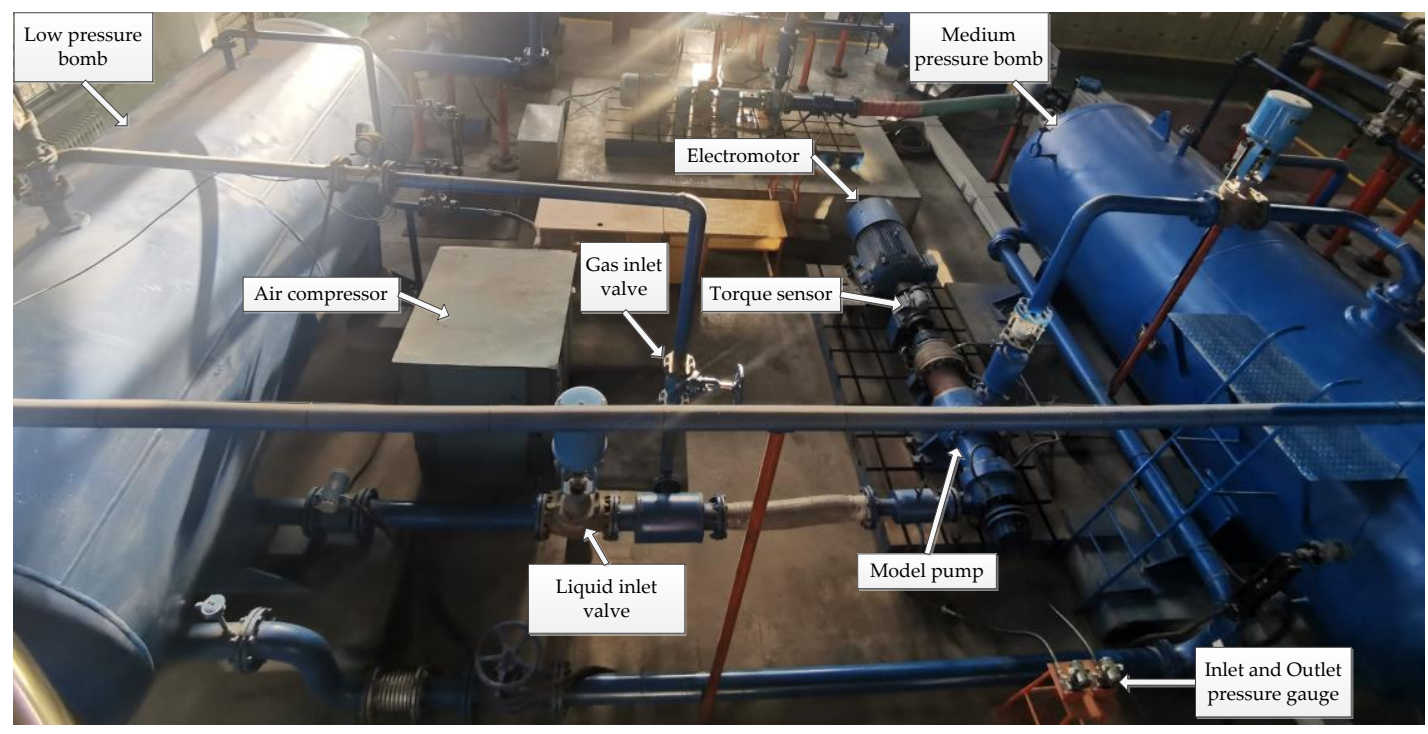

(b)

Figure 3. Gas-liquid two-phase flow pump performance test bench. (a) The schematic diagram of the gas-liquid two-phase flow pump performance test bench. 1,4,15,16,18-Pressure gauge; 2-Electromagnetic flow meter; 3-Liquid inlet valve; 5-Low pressure bomb; 6-Gas valve; 7-Air compressor; 8-Air compressor control room; 9-Flowmeter; 10-Gas inlet valve; 11-Electromotor; 12-Coupling; 13-Torque sensor; 14-Model pump; 17-Medium pressure bomb. (b) Picture of the test bench. 




(a)

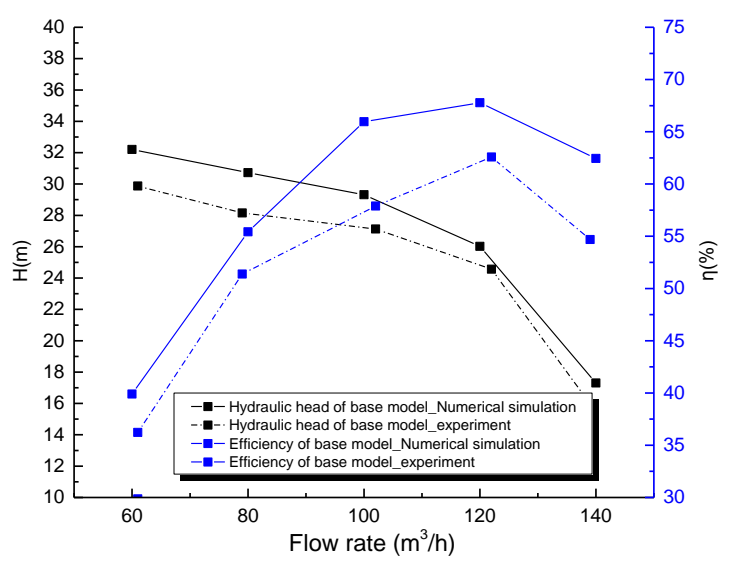

(b)

Figure 4. Hydraulic performance curves of a multiphase pump with different flow rates. (a) Inlet gas volume fraction $(\mathrm{IGVF})=30 \%$ and $(\mathbf{b}) \mathrm{IGVF}=50 \%$.

Figure 5 shows the gas volume fraction (GVF) distribution of the meridional surface in the impeller passage with different flow rates. The meridional surface with different flow rates had the same horizontal and vertical scales. With the increasing of the flow rate, the aggregation area of gas in the flow passage gradually moved from the inlet to the outlet of the impeller. The maximum gas volume fraction in the flow passage decreased with the increase in flow rate, which indicates that the increase in flow was conducive to the outflow of gas. The gas accumulation occurred near the middle area of the flow passage under the working condition of $0.6 Q_{0}$, and the gas aggregation was more serious than under other working conditions. The accumulation of the gas phase in the flow passage partly occurred near the hub of the impeller; when the gas phase accumulated to a certain extent, it was more likely to cause the unstable operation condition under the $0.6 Q_{0}$ condition than other conditions. The entrainment effect of the liquid phase on the gas phase decreased with the decrease of flow rates. The density of the gas phase was lower than that of the liquid phase, so the centrifugal force acted less in the gas phase than the liquid phase, and the pressure from the liquid phase to the gas phase was larger. Therefore, the gas was concentrated near the hub of the impeller outlet. With the increase in flow rate, the gas accumulation decreased, but the accumulation area was still near the hub area, which was mainly caused by the expelling effect due to the density difference of the gas and liquid phases. The expelling effect means the effect of the gas phase moving with liquid phase. The energy of the fluid increases with the increasing of the flow rate, so the ability of the low-density phase to follow the high-density phase increased. The larger the flow rate, the more obvious the expelling effect. The increase of the expelling effect, the difference in density and pressure in the gas-liquid two-phase caused the gas phase accumulated to the outlet area near the hub of the impeller passage more easily. 




(a) $0.6 \mathrm{Q}_{0}$

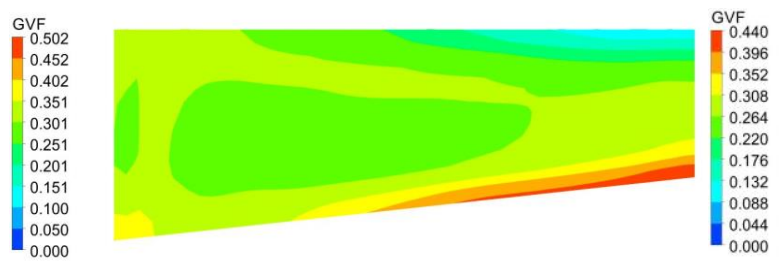

(c) $\mathrm{Q}_{0}$

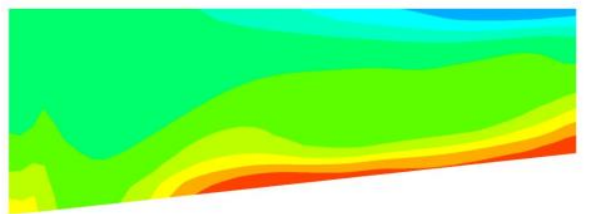

(b) $0.8 \mathrm{Q}_{0}$

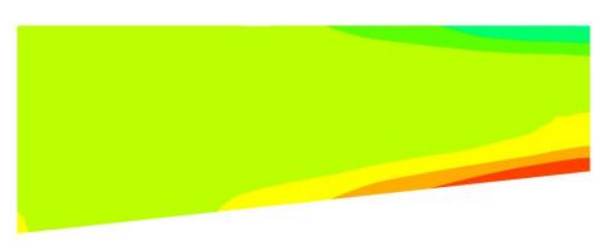

(d) $1.2 Q_{0}$

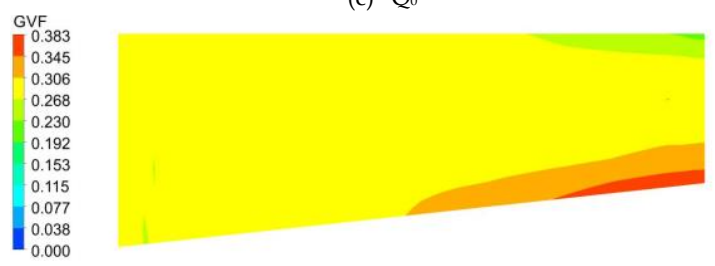

(d) $1.4 \mathrm{Q}_{0}$

Figure 5. Gas volume fraction (GVF) distribution of the meridional surface in the impeller passage at different flow rates.

The hydraulic performance of the multiphase pump with inlet gas volume fractions of $0.3,0.4$, 0.5, 0.6, and 0.7 under the design working condition are shown in Figure 6. The dimensionless head coefficient, defined in Equation (12), was used to describe the change in head with increasing inlet gas volume fraction. The head coefficient and efficiency of the multiphase pump both decreased with the increasing inlet gas volume fraction. In other words, the boosting capacity of the impeller and the performance of the multiphase pump decreased with the increasing inlet gas volume fraction.

The head coefficient $\Psi$ is defined as

$$
\Psi=\frac{2 \mathrm{gH}}{u_{2}^{2}}
$$

where $u_{2}$ is the circumferential velocity of the impeller shroud and $g$ is the local gravity acceleration; their units are $\mathrm{m} / \mathrm{s}$ and $\mathrm{m} / \mathrm{s}^{-2}$, respectively.

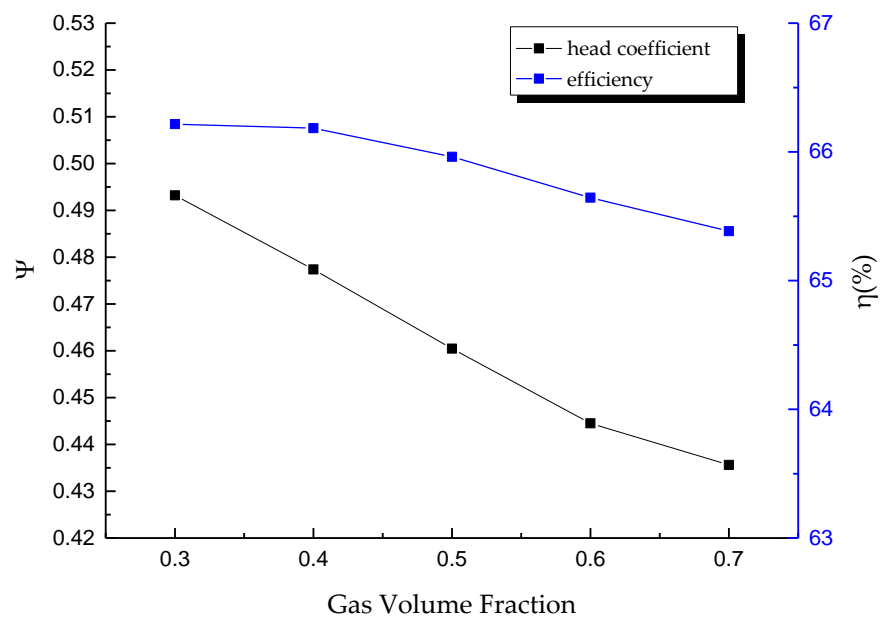

Figure 6. Hydraulic performance of a multiphase pump with different inlet gas volume fractions. 
The gas distributions at $10 \%$ of the blade height with inlet gas volume fractions of $0.3,0.4$, $0.5,0.6$ and 0.7 in the flow passage of impeller and diffuser were determined as shown in Figure 7. With the increasing of inlet gas volume fraction, the gas and liquid phases near the pressure side of the impeller blade mixed well. However, the gas accumulation phenomenon became more serious and the accumulation area increased accordingly in the region close to the suction side of blade, but the location of gas accumulation was basically maintained, which was concentrated on the suction side of the impeller near the hub. This was the position at which the pressure gradient was the largest in the impeller passage. Coupled with the influence of centrifugal force and pressure, the gas is more susceptible to gathering in areas of lower pressure and a small radius. With the increase in the gas volume fraction, the gas gradually diffused from the outlet of the backside to the inlet along the hub. Figure 7 also shows that the rotor-stator interaction between the impeller and diffuser strongly influenced the distribution of the gas phase in the diffuser flow passage. It is found in the figure that there is a certain rule in the distribution of gas phase. The main manifestation is that there were obvious changes between the interference region and the non-interference region of the stator and rotor cascades. The gas volume fraction of the diffuser flow passage was relatively large in the area adjacent to the blade of impeller, as shown in the black wireframe section of Figure 7 . Since the gas phase in the diffuser was not affected by the centrifugal force, the distribution of the gas phase in the diffuser was relatively dispersed.

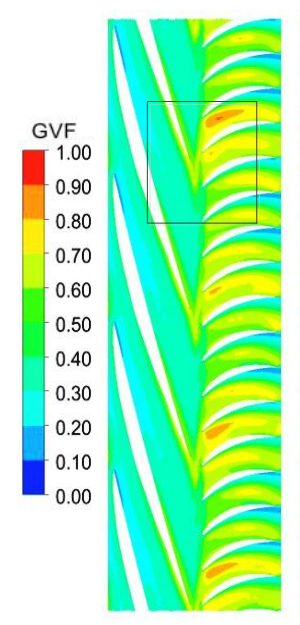

$\mathrm{IGVF}=0.3$

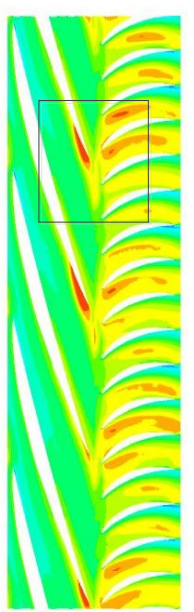

$\mathrm{IGVF}=0.4$

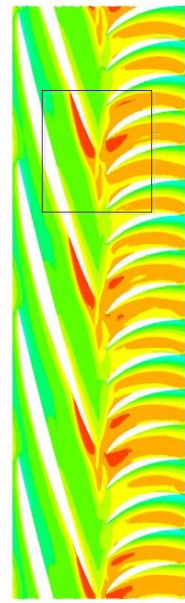

IGVF $=0.5$

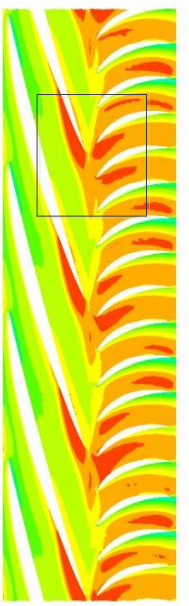

$\mathrm{IGVF}=0.6$

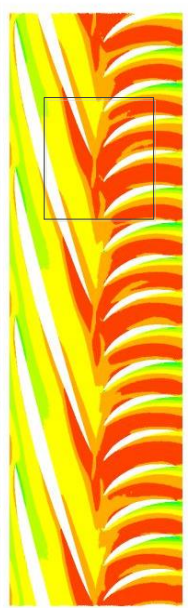

IGV F= 0.7

Figure 7. Distribution of gas at $10 \%$ of the blade height with different inlet gas volume fractions (IGVFs) under the design working conditions.

\section{Optimization Design Scheme and Results Analysis}

\subsection{Optimization Design Scheme}

The thickness variation rule of the 791 airfoil was applied to the blade thickening of the multiphase pump; the thickening law is shown in Table 7. The 791 airfoil is shown in Figure 8.

Table 7. The thickening law of a 791 airfoil; $x$ is the length from the inlet of the airfoil, $l$ is the chord length of the airfoil, $\delta$ denotes the thickness corresponding to the $x$ position and $\delta_{\max }$ is the maximum thickness of the airfoil.

\begin{tabular}{ccccccccccccccc}
\hline$x / l$ & 0 & 0.05 & 0.075 & 0.1 & 0.2 & 0.3 & 0.4 & 0.5 & 0.6 & 0.7 & 0.8 & 0.9 & 0.95 & 1 \\
\hline$\delta / \delta_{\max }$ & 0 & 0.296 & 0.405 & 0.489 & 0.778 & 0.92 & 0.978 & 1 & 0.883 & 0.756 & 0.544 & 0.356 & 0.2 & 0 \\
\hline
\end{tabular}




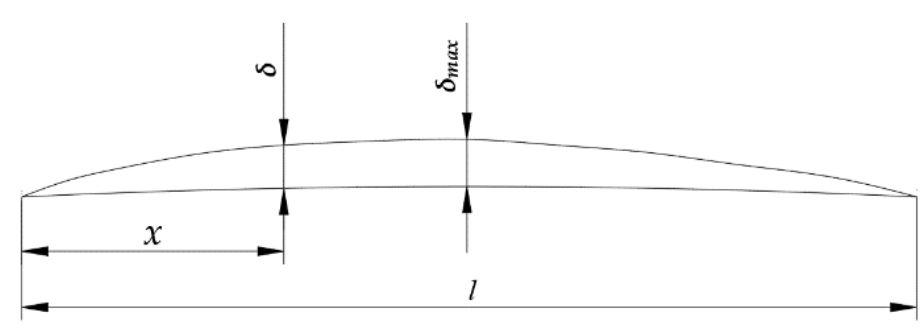

Figure 8. The 791 airfoil.

The maximum thickness of the airfoil $\left(\delta_{\max }\right)$ at the hub was determined according to the strength condition [44], usually estimated roughly according to

$$
\delta_{\max }=(0.012-0.015) \mathrm{D} \sqrt{\mathrm{H}},
$$

where $\mathrm{D}$ is the diameter of the impeller shroud of the multiphase pump; its unit is $\mathrm{m}$.

Practice has proven that the thickness of the blade calculated according to the above equation is often too thick, which is not conducive to improving the performance of the pump [45]. The thickness is related to the chord length, and the relative thickness of the hub section is generally calculated as

$$
\bar{\delta}_{h u b}=\frac{\delta}{l}(10 \%-15 \%),
$$

and the relative thickness of the shroud section is calculated as

$$
\bar{\delta}_{\text {shroud }}=\frac{\delta}{l}(2 \%-5 \%)
$$

The hub is the thickest part generally, and the thickness varies linearly from the hub to the shroud. The maximum thicknesses of the hub and shroud for the basic model were selected as 9 and $5 \mathrm{~mm}$, respectively. The airfoil from the hub to the shroud of the blade is shown in Figure 9.

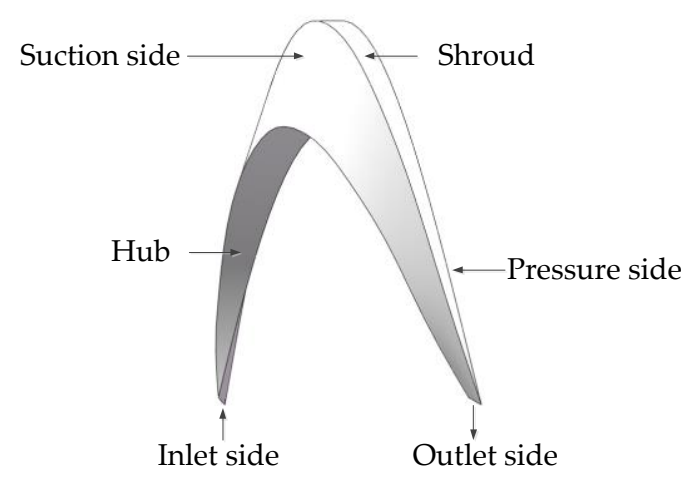

Figure 9. The frame of the helical-axial blade.

To investigate the effects of the thickness variation in the airfoil from the hub to the shroud of the blade on the hydraulic performance of the multiphase pump, the thickness ratio coefficient $\xi$ is defined as

$$
\xi=\frac{\delta_{h \max }}{\delta_{s \max }}
$$

where $\delta_{h \max }$ and $\delta_{s} \max$ denote the maximum thicknesses in the airfoil at the side of the hub and shroud, respectively, and $\xi$ indicates the change degree of the blade thickness from hub to shroud.

The thickness ratio coefficients $\xi$ were all greater than 1 according to the usual regular thick airfoil. To investigate the influence of the multiphase pump when $\xi \leq 1$, different thickening schemes were selected on the basis of ensuring the strength of the blade, as shown in Figure 10. The thickness ratio 
coefficient was selected to range from 0.8 to $1.8 ; \delta_{h \max }$ was set to $7.5,8,8.5$, and $9 \mathrm{~mm}$, representing schemes 1 to 4 , respectively; and $\xi=1.8$ was the thickening scheme of the basic model. As shown in Figure 10, the maximum thickness of the hub on the same curve was the same; the $x$ coordinates from left to right represent the increase in the maximum thickness at the side of shroud and the vertical axis represents different thickness ratio coefficients.

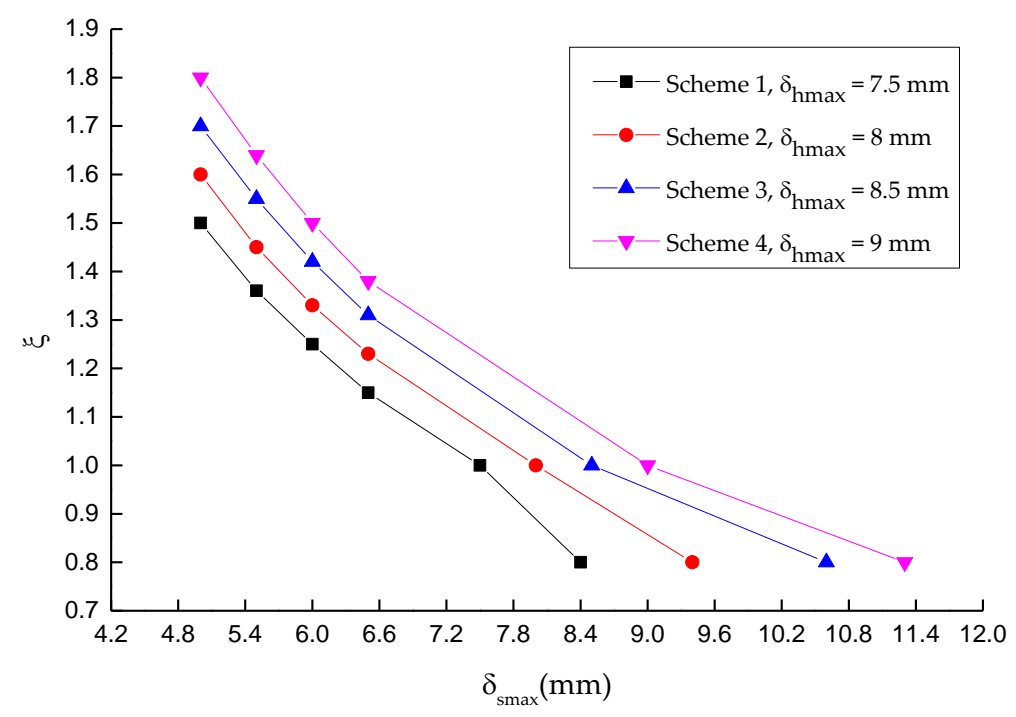

Figure 10. Thick airfoil schemes.

The main step of the optimization design of the impeller is: in order to investigate the influence of the thickness variation from hub to shroud on the characteristics of multiphase pump, the thickness ratio coefficient was defined. The variables that affect the thickness ratio coefficient were the maximum thickness of the impeller hub and the maximum thickness of the shroud. Different thickness ratio coefficients were obtained by taking different values for these two key variables, so that the thickness coefficient ratio contained different ranges from 0.8 to 1.8. By comparing the hydraulic performance of the multiphase pump with different schemes, such as the head coefficient and efficiency, analyzing the internal flow condition and the aggregation degree of gas phase in the impeller passage of different schemes, the rule and relation between the mixture transportation characteristics of the multiphase pump and the value of coefficient was finally summarized. By adjusting the value of the thickness ratio coefficient and analyzing the simulation results of different thickness ratio coefficient schemes, the optimization design of the impeller was achieved, the impeller model, which had higher hydraulic performance, could be found. Finally, a multiphase pump with higher mixture transportation performance would be obtained.

\subsection{Results Analysis}

Figure 11 shows the changes trend of the head coefficient $\Psi$ and efficiency $\eta$ with different thickness schemes under the designed working condition when the inlet gas volume fraction was 0.5. Figure 11 shows that when $\delta_{\text {hmax }}$ remained unchanged and $\delta_{\text {smax }}$ increased-i.e., reducing the thickness ratio coefficient $\xi$-the head coefficient and efficiency increase accordingly in every scheme. The data of the four schemes show the same trend, which is helpful for summarizing the relationship between the hydraulic performance of the multiphase pump and the thickness ratio coefficient. We concluded that the reduction in the thickness ratio coefficient $\xi$ with the same $\delta_{\text {hmax }}$ was conducive to improving the boosting capability and efficiency of multiphase pumps. The head coefficient of the modification multiphase pump was $5.8 \%$ higher in comparison to the base pump, while the efficiency was $3.1 \%$ higher than that of the base pump. 


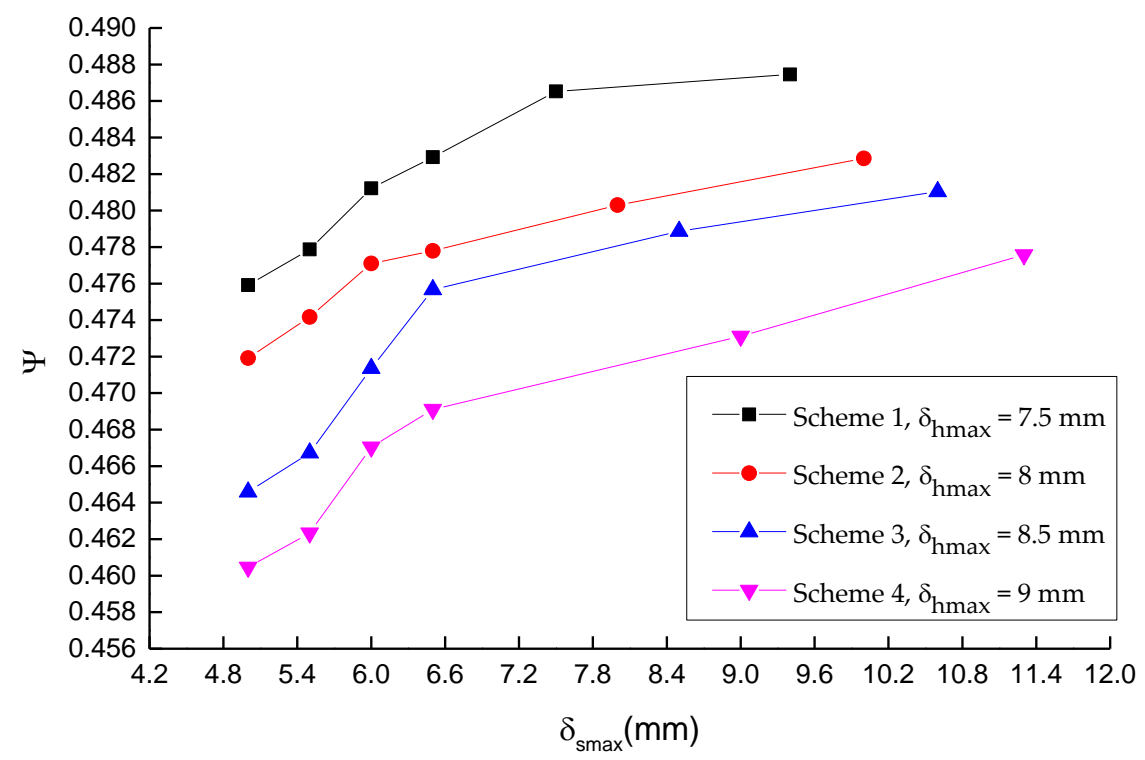

(a)

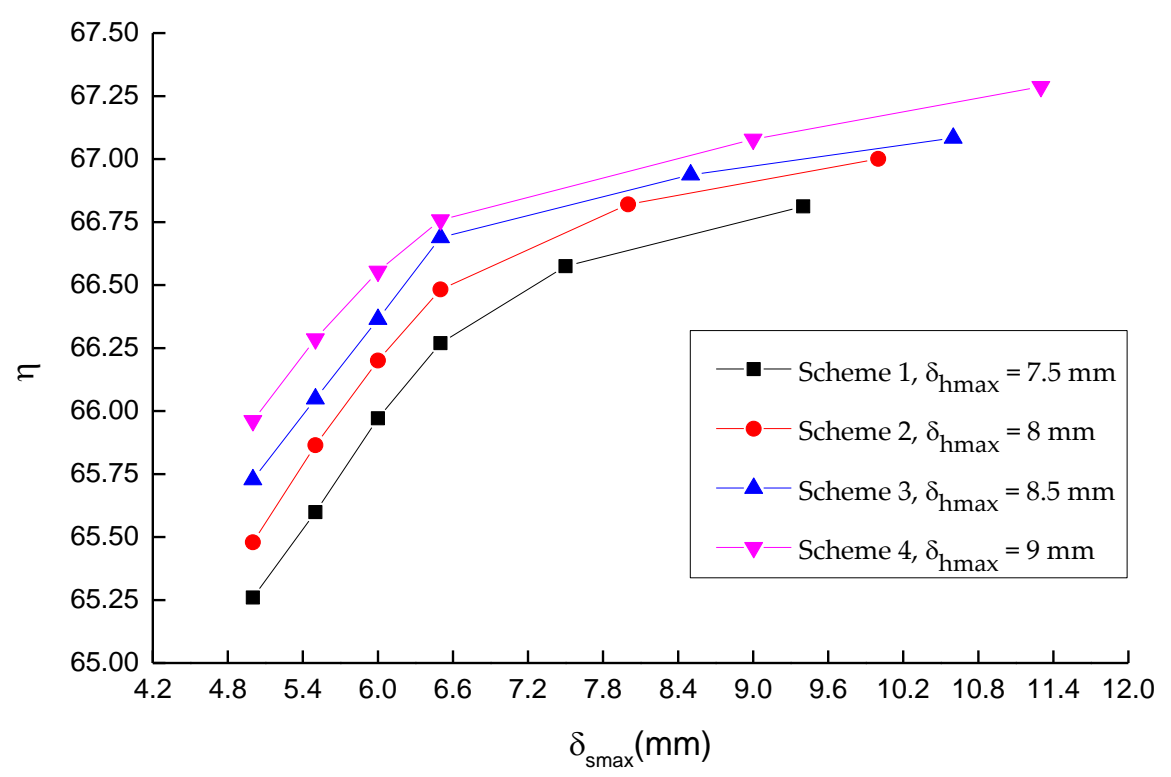

(b)

Figure 11. The relationship of the (a) head coefficient and (b) efficiency with different thickness schemes.

Figure 12 shows the main area of gas accumulation in the flow passage, which is the outlet of the impeller suction side near the hub; the mixing of the gas-liquid two-phase in the other regions of the impeller was better than the accumulation area. Therefore, $10 \%$ of the blade height surface was selected to analyze the distribution of the gas-liquid two-phase in the flow passage. 


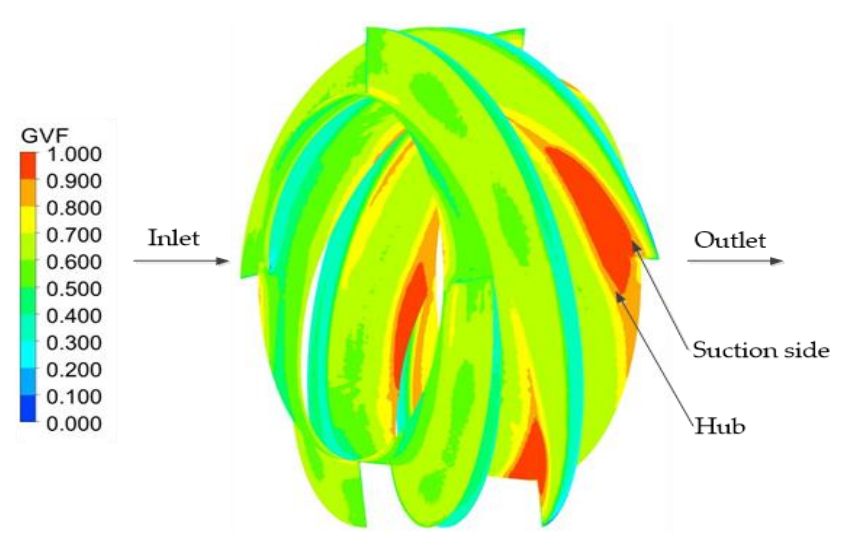

Figure 12. The accumulation of gas in the flow passage.

Figure 13 shows the distribution of gas at 10\% of the blade height with different thickness schemes when the inlet gas volume fraction was 0.5 . Since too many figures will occupy a large space in the article, we selected only the distribution of gas with scheme 1 and scheme 4 in Figure 13. As the gas phase mainly accumulates at the hub and gradually decreased from the hub to the shroud, the volume distribution of gas phase at $10 \%$ of the blade height near the hub was selected for analysis. Figure 13 shows that, when keeping $\delta_{h \max }$ unchanged and increasing $\delta_{\text {smax }}$, which was equivalent to the process of decreasing the thickness ratio coefficient $\xi$, the accumulation phenomenon of gas also improved. The gas mainly accumulated at the blade outlet of the suction side near the hub. The gas-liquid two-phase flow near the pressure side was always well mixed, and thus no significant separation phenomenon occurred.



(a)

Figure 13. Cont. 


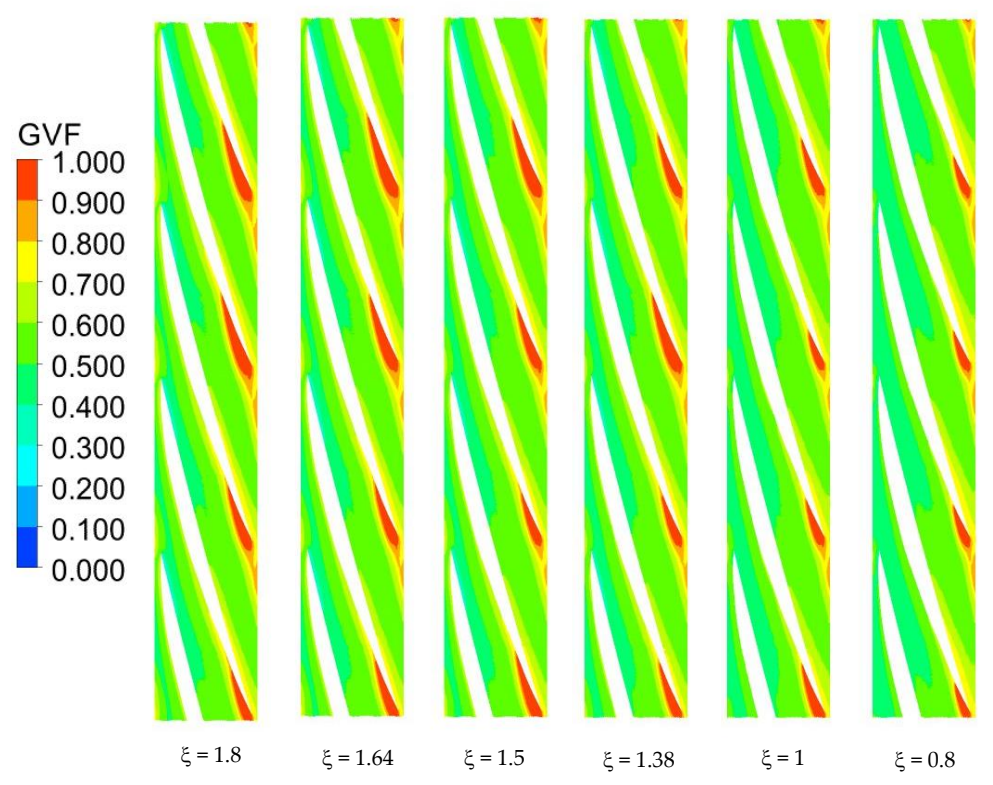

(b)

Figure 13. The gas distribution at $10 \%$ of the blade height under different thickness schemes with an inlet gas volume fraction of 0.5 . (a) Scheme 1 with $\delta_{h \max }=7.5 \mathrm{~mm}$ and (b) scheme 4 with $\delta_{h \max }=9 \mathrm{~mm}$.

With the linear thickening of the blade from hub to shroud, different values of the thickness ratio coefficient $\xi$ corresponded to different profile lines of the blade; the profile of the pressure side was the same when the thickness ratio coefficient $\xi$ changes. The changes in the airfoil thickness impacted the inclination degree of the blade backside. During the rotation of the impeller, the pressure side and the suction side of the blade acted with force on the two-phase medium. The force will change due to the changes in the inclination degree following the change in thickness. The larger the thickness ratio coefficient $\xi$, the more inclined the backside of the blade is toward the shroud. Under the coupling action of centrifugal force and the force acted by the suction side of the blade, the high-density liquid phase becomes closer to the area near the shroud and the low-density gas phase moves closer to the hub. When the thickness ratio coefficient $\xi \geq 1$, the radial component direction of the force points to the shroud along the hub, which was exerted on the gas-liquid two-phase medium by the suction side of the blade. This is consistent with the direction of the centrifugal force and provides an additional promotion of the separation of the gas-liquid two-phase flow. Thus, the separation of gas-liquid two-phase flow is more serious and causes the hydraulic head of the multiphase pump to decrease. Conversely, when the thickness ratio coefficient $\xi<1$, the direction of the force acts on the gas-liquid two-phase as the suction side of the blade points to the hub along the shroud. The direction of the force is opposite to the centrifugal force, which reduces part of the separation inductive force. Therefore, on the premise that the hub thickness meets the strength requirement and the shroud thickness meets the process conditions, reducing the thickness ratio coefficient $\xi$ can improve the two-phase transportation performance of the multiphase pump.

Figure 14 shows the schematic diagram of force on the fluid particle in the axial section of the impeller with the thickness ratio coefficient $\xi>1$ and $\xi<1$. In the figure, $F_{c}$ is the centrifugal force, $F_{p}$ represents the pressure gradient force, which is caused by the pressure gradient at the side of shroud and hub, $F_{t}$ denotes the force applied by the suction side of the impeller-the direction of $F_{t}$ is perpendicular to the suction side-and $F_{t x}$ is the radial component force of $F_{t}$. The separation inductive force was caused mainly because $F_{c}>F_{p}$. The direction of $F_{t x}$ changed due to the reduction of thickness ratio coefficient; when the thickness ratio coefficient $\xi<1$, the direction of $F_{t x}$ pointed to the hub along the shroud, which reduced the difference between the $F_{c}$ and $F_{p}$-i.e., it reduced part of the separation inductive force. 




Figure 14. Schematic diagram of force on fluid particles in the axial section of the impeller.

According to the gas distribution at $10 \%$ of the blade height, we concluded that the aggregation of gas mainly occurs at the blade outlet of the suction side near the hub. To express the accumulation phenomenon of gas in the flow passage with different thickening schemes more intuitively, the area starting from half the axial length $(e)$ of the impeller in the axial direction to the outlet in each thickening scheme was selected as the analysis area, where the accumulation phenomenon was the most serious in the impeller and represented the accumulation degree of the gas in the flow passage, as shown in Figure 15.

Inlet of the impeller

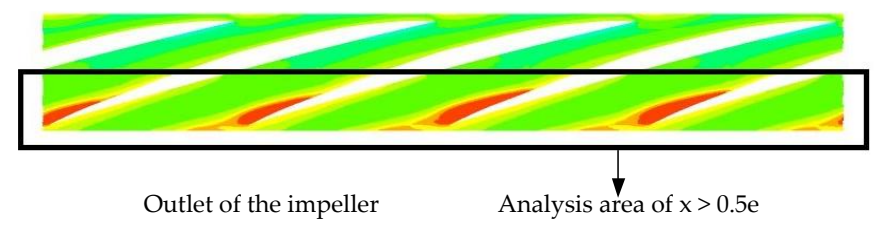

Figure 15. Analysis area of the gas distribution at $10 \%$ of the blade height.

The governing equations were discretized using a finite volume method (FVM), which first involves discretizing the spatial domain using a mesh. The mesh was used to construct finite volumes, which were used to conserve relevant quantities [46]; the conceptual diagram of the finite volume method is shown in Figure 16. The scalar variables such as mass, momentum and phase-volume fraction are taken at the cell center [47]. The gas distribution and grid area date of all grids at $10 \%$ of the blade height can be derived from the center of the cell using the internal point method. Firstly, the gas volume fraction and area data whose axial coordinates were greater than half the axial length of the impeller were filtered. Then, the grid area data of the region with a volume fraction greater than 0.7 were filtered and summed.

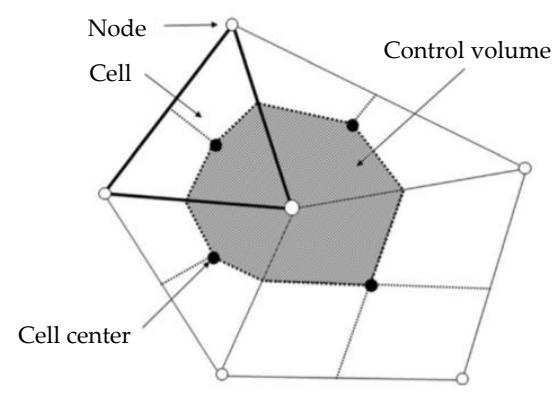

Figure 16. Conceptual diagram of finite volume method. 
In order to represent the degree of gas accumulation in the analysis area more intuitively, the aggregation degree $\lambda$ was introduced in Equation (17). The larger the value, the more serious the gas accumulation is in this area:

$$
\lambda=\frac{S_{v o f}>0.7}{S_{x>0.5 e}}
$$

where $S_{x}>0.5 e$ represents the total grid area of the analysis area, whose axial coordinate is greater than one-half axial length of the impeller; $e$ is the axial length of impeller and $S_{v o f}>0.7$ is the total grid area of the region with a gas volume fraction greater than 0.7 in the analysis region.

Figure 17 shows the variation in the aggregation degree $\lambda$ with different thickness schemes. When $\delta_{\text {hmax }}$ is unchanged, the aggregation degree $\lambda$ decreases with the decrease in thickness ratio coefficient $\xi$, which indicates that the aggregation phenomenon of gas is improved in the flow passage with the reduction in thickness ratio coefficient $\xi$. This is contrasting to the change trend of the hydraulic performance of the multiphase pump; i.e., the reduction of the hydraulic performance of the multiphase pump is directly related to the aggregation degree $\lambda$ in the flow channel. The value of $\lambda$ is inversely related to the hydraulic performance in every thickness scheme. The smaller the value of the aggregation degree $\lambda$, the better the hydraulic performance of the multiphase pump. The gas accumulation in the flow passage of the multiphase pump can be improved by appropriately reducing the thickness ratio coefficient $\xi$. The aggregation of the modification multiphase pump is $30.3 \%$ lower in comparison to the base pump, which means the aggregation phenomenon of gas phase in the impeller passage is improved after modification.



Figure 17. The variation in the aggregation degree with different thickness schemes.

If complete separation does not occur in $90 \%$ of the chord length area from the inlet to the outlet in the flow passage, the airfoil of the impeller can be regarded as a super-separated airfoil. The impeller model with scheme 1 of $\xi=0.8$ can delay the completely separated area beyond the region with a chord length greater than $90 \%$. This basically solves the problem of preventing the separation of the gas-liquid phase in the middle and rear parts of the flow passage. The airfoil of the multiphase pump in this scheme can be regarded as a super-separated airfoil.

Figure 18 shows the turbulent kinetic energy of the meridional surface with different thickness schemes. We also selected the turbulent kinetic energy with scheme 1 and scheme 4 in Figure 18 to save space in the article. The gradient of the turbulent kinetic energy at the inlet of the impeller was relatively large, because the fluid entering the passage of the impeller impacted the surface of the blade, resulting in unstable flow at the inlet. The flow was more stable in the middle area of the runner. The turbulent kinetic energy and gradient near the hub at the outlet of the impeller were higher than 
in other regions, which was consistent with the gas accumulation areas in the passage of impeller. Combined with Figure 17, it can be seen that the change trend in the turbulent kinetic energy gradient at the impeller outlet and the aggregation degree $\lambda$ of each scheme were consistent with the change in the thickness ratio coefficient $\xi$. When the $\delta_{\text {hmax }}$ was the same, the higher the thickness ratio coefficient $\xi$ was, the larger the turbulent kinetic energy gradient and aggregation degree. The accumulation of gas caused additional energy loss in the flow passage with the increase of aggregation degree. This reveals why the head coefficient $\Psi$ and efficiency $\eta$ increase with the decreases of thickness ratio coefficient $\xi$. The gas phase aggregation phenomenon in the flow passage of impeller improved and the energy loss decreased, while the thickness ratio coefficient $\xi$ decreased, so the hydraulic performance of the multiphase pump improved. Considering the technological factors in the actual processing and the crowding-out effect of the blade, the thickness ratio coefficient in the present study could be set as low as 0.8 .

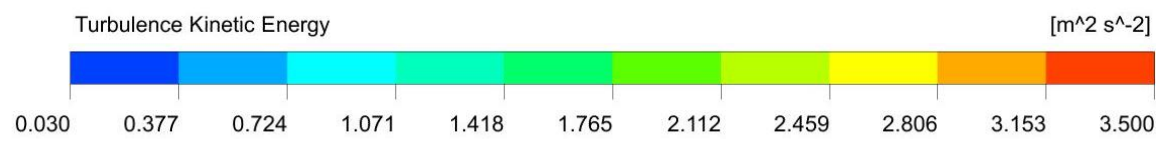

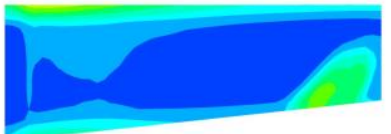

$\xi=1.5$

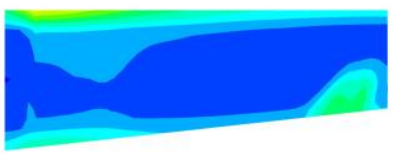

$\xi=1.36$

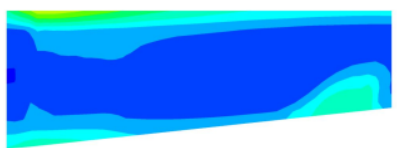

$\xi=1.25$



$\xi=1.15$

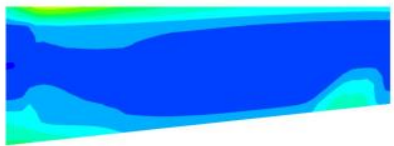

$\xi=1$



$\xi=0.8$

(a)



$\xi=1.8$



$\xi=1.64$

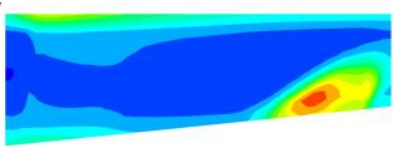

$\xi=1.5$



$\xi=1.38$

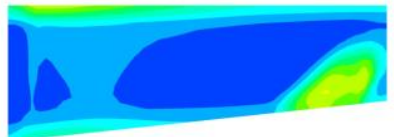

$\xi=1$

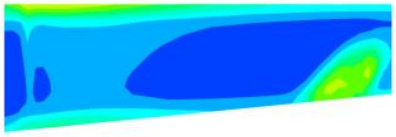

$\xi=0.8$

(b)

Figure 18. Turbulent kinetic energy of meridional surface with different thickening schemes. (a) Scheme 1 with $\delta_{\text {hmax }}=7.5 \mathrm{~mm}$ and (b) scheme 4 with $\delta_{\text {hmax }}=9 \mathrm{~mm}$.

\section{Conclusions}

A single compression unit was designed to investigate the mixture transportation characteristics of the gas-liquid two-phase flow in a helical-axial pump. By comparing the numerical simulation 
results and experimental results, the accuracy of the numerical simulation method with the Eulerian multiphase model and the shear stress transport (SST) $k$-w turbulence model on helical-axial multiphase pumps was validated. The head coefficient and efficiency of a multiphase pump with different schemes were obtained and the flow characteristics were analyzed. The following conclusions could be drawn:

(1) As the head coefficient $\Psi$ and efficiency $\eta$ increase, the aggregation degree of gas $\lambda$ decreased with the decreasing of the thickness ratio coefficient $\xi$ while the hub thickness was maintained. The head coefficient of the modification multiphase pump was $5.8 \%$ higher in comparison to the base pump, while the efficiency was 3.1\% higher than that of the base pump; the optimal model in the research was the model of scheme 1 with $\xi=0.8$.

(2) When the maximum thickness of the hub was unchanged, the reduction of the thickness ratio coefficient $\xi$ decreased some of the separation inductive force of the gas-liquid two-phase flow, which is conducive to inhibiting the separation of the gas-liquid two-phase, thus improving the gas-liquid two-phase transportation performance of multiphase pumps.

(3) A super-separated airfoil was defined and found to be $\xi=0.8$ in scheme 1 , which was able to delay the separation area of the gas and liquid two-phase flow beyond the region with a chord length greater than $90 \%$. The aggregation degree of this model was the lowest, which was $30.3 \%$ lower in comparison with the base pump.

(4) The change trend in the gradient of turbulent kinetic energy in the flow passage of the impeller was related to the distribution of gas. The gas accumulation region was similar to the area where the gradient of turbulent kinetic energy was large, and so the energy loss could be interpreted. The accumulation phenomenon of gas phase became more and more obvious along the hub side from the inlet to the outlet, especially at the outlet area.

(5) The research on the influence of the thickness ratio coefficient on the multiphase pump provided some reference value for the modification design of impellers. In future work, visual observation techniques of the internal flow field can be adopted to provide visual evidence for the modification of the multiphase pump.

Author Contributions: W.H. conceived and designed the research, X.L. drafted the article, Y.S. completed the English check, M.S. processed the data, R.L. was the leader of the research team, and Y.Z. organized the layout of the manuscript. All authors have read and agreed to the published version of the manuscript.

Funding: This study was supported by the National Key R\&D Program Projects of China [Grant no. 2018YFB0606100], and the National Natural Science Foundation of China [Grant no. 51669012, 51966010].

Conflicts of Interest: There are no known conflicts of interest.

\section{References}

1. Hua, G.; Falcone, G.; Teodoriu, C. Comparison of multiphase pumping technologies for subsea and downhole applications. Oil Gas Facil. 2012, 1, 36-46. [CrossRef]

2. Cao, F.; Shu, P.C. Technology and research progress of twin-screw type multiphase pump. China Petrol. Mach. 1999, 3, 49-52.

3. Shao, C.L.; Li, C.Q.; Zhou, J.F. Experimental investigation of flow patterns and external performance of a centrifugal pump that transports gas-liquid two-phase mixtures. Int. J. Heat Fluid Flow 2018, 71, 460-469. [CrossRef]

4. Salis, J.D.; Marolles, C.D.; Falcimaigne, J. Multiphase pumping-operation and control. In Proceedings of the SPE Annual Technical Conference and Exhibition 1996, Denver, CO, USA, 6-9 October 1996.

5. Li, Q.P.; Xue, D.S.; Zhu, H.W.; Li, Z.F. Research on hydraulic design concept of an helical-multiphase pump and its experimental studies on performances. J. Eng. Therm. 2005, 26, 84-87.

6. Dal Porto, D.F. Multiphase-pump trails demonstrate practical applications for the technology. SPE Prod. Facil. 1997, 12, 159-164. [CrossRef] 
7. Samsudin, Z.B.; Ajmi, S.M.; Qudaihy, D.S. Production enhancement using a multiphase helico-axial pump in a remote hilly-terrain oil field in Saudi Arabia. In Proceedings of the SPE Saudi Arabia Section Technical Symposium and Exhibition 2012, Al-Kahobar, Saudi Arabia, 8-11 April 2012.

8. Saadwi, H. An overview of multiphase pumping technology and its potential application for oil fields in the gulf region. In Proceedings of the International Petroleum Technology Conference 2007, Dubai, UAE, 4-6 December 2007.

9. Gruehagen, H.; Lim, D. Subsea separation and boosting-An overview of ongoing projects. In Proceedings of the 2009 SPE Asia Pacific Oil and Gas Conference and Exhibition, Jakarta, Indonesia, 4-6 August 2009.

10. Davis, B.E.; Kelly, C.; Kierulf, K. BP King-Deep multiphase boosting made possible. In Proceedings of the 2009 Offshore Technology Conference, Houston, TX, USA, 4-7 May 2009.

11. Magrini, K.L.; Sarica, C.; Al-Sarkhi, A. Liquid entrainment in annular gas/liquid flow in inclined pipes. SPE J. 2012, 17, 617-630. [CrossRef]

12. Cioncolini, A.; Thome, J.R. Entrained liquid fraction prediction in adiabatic and evaporating annular two-phase flow. Nucl. Eng. Des. 2012, 243, 200-213. [CrossRef]

13. Abelsson, C.; Busland, H.; Henden, R. Development and testing of a hybrid boosting pump. In Proceedings of the Offshore Technology Conference, Houston, TX, USA, 2-5 May 2011.

14. Ma, X.J.; Qu, X.; Xiao, X.J. Three-dimensional modeling of lamina on axial gas-oil multiphase pump. Drain. Irrig. Mach. 2008, 26, 15-17.

15. Ma, X.J.; Wang, Z.; Zhang, M.Z. Numerical simulation and flow analysis on compression stage of oil-gas mixture pump based on CFX software. Oil Field Equip. 2011, 40, 32-36.

16. Yu, Z.Y.; Cao, S.L.; Wang, G.Y. CFD analysis of the air-water bubbly flow in a multiphase rotodynamic pump impeller. J. Eng. Thermophys. 2007, 28, 46-48.

17. Li, Q.P.; Xue, D.W.; Li, Z.F. Optimize of the helico-axial multiphase pump prototype and its experimental studies on performances. J. Eng. Thermophys. 2004, 25, 962-964.

18. Cao, S.L.; Peng, G.Y.; Yu, Z.Y. Hydrodynamic design of rotodynamic pump impeller for multiphase pumping by combined approach of inverse design and CFD analysis. J. Fluids Eng. 2005, 127, 330-338. [CrossRef]

19. Zhang, J.Y.; Zhu, H.W. Numerical simulation of gas-liquid two-phase with blade-type multiphase pump. Pump Technol. 2010, 5, 32-39.

20. Kong, X.L.; Lv, Y.; Gao, J.W. Research on multi-stage compressible simulation of helico-axial multiphase pump. China Petrol. Mach. 2016, 44, 77-81.

21. Zhang, J.Y.; Zhu, H.W.; Xu, B.G. Methods for improving gas and liquid phases mixing in impeller with high gas void fraction. J. Drain. Irrig. Mach. Eng. 2012, 30, 641-645.

22. Kim, J.D.; Lee, H.C. Reliability verification of the performance evaluation of multiphase pump. Eng. Technol. Int. J. Mech. Mechatron. Eng. 2014, 8, 1985-1989.

23. Suh, J.W.; Kim, J.H.; Choi, Y.S. A study on numerical optimization and performance verification of multiphase pump for offshore plant. J. Power Energy 2017, 231, 382-397. [CrossRef]

24. Ma, X.J.; Li, X.K.; Han, W.; Wang, N. Optimization design of multiphase pump using combined orthogonal design and neural network. J. Lanzhou Univ. Technol. 2011, 37, 37-41.

25. Shi, Y.; Zhu, H.W.; Yin, B.B. Numerical investigation of two-phase flow characteristics in multiphase pump with split vane impellers. J. Mech. Sci. Technol. 2019, 33, 1-11. [CrossRef]

26. Lee, H.C.; Kim, Y.H. Performance Improvement of Multiphase Pump for Offshore Plants through Optimization Technique. In Proceedings of the 6th International Symposium on Fluid Machinery and Fluids Engineering, Wuhan, China, 22-25 October 2014.

27. Shi, G.T.; Wang, Z.W. Pressurization performance of different areas within multiphase pump impeller. J. Drain. Irrig. Mach. Eng. 2019, 37, 13-17.

28. Shi, G.T.; Luo, K.; Wang, Z.W. Study on load distribution characteristics of axial flow multiphase pump. Water Resour. Power 2018, 36, 175-178.

29. Liu, M.; Cao, S.; Cao, S.L. Numerical analysis for interphase forces of gas-liquid flow in a multiphase pump. Eng. Comput. 2018, 35, 2386-2402. [CrossRef]

30. Sher, F.; Sajid, Z.; Tokay, B.; Khzouz, M.; Sadiq, H. Study of gas-liquid mixing in stirred vessel using electrical resistance tomography. Asia Pac. J. Chem. Eng. 2016, 11, 855-865. [CrossRef]

31. Zhang, L.P.; Chang, X.H. Mesh Generation Techniques in Computational Fluid Dynamics; Science Press: Beijing, China, 2017; pp. 10-12. 
32. Dong, L.; Liu, H.L. Detailed CFD Numerical Calculation Example of Vane Pump; China Machine Press: Beijing, China, 2015; p. 120.

33. Yuan, S.F. The Characteristics of Gas-Liquid Mixed Transportation in Helical Axial-Flow Multiphase Pump. Master's Thesis, Lanzhou University of Technology, Lanzhou, China, May 2019.

34. Ge, Z.G. Numerical simulation of internal flow characteristics of centrifugal pump under the condition of bubble inflow. In Proceedings of the 11th National Conference on Hydraulic Machinery and Its Systems, Beijing, China, 19-22 October 2018.

35. Wang, G.Y.; Huo, Y.; Zhang, B. Evaluation of Turbulence Models for Predicting the Performance of an Axial-Flow Pump. Trans. Beijing Inst. Technol. 2009, 29, 309-313.

36. Yu, Z.Y.; Zhang, Q.Z. Numerical analysis of gas-liquid mixed transport process in a multiphase rotodynamic pump. IOP Conf. Ser. Earth Environ. Sci. 2012, 15, 2062. [CrossRef]

37. Yi, S.; Zhu, H.W.; Zhang, J.Y. Experiment and numerical study of a new generation three-stage multiphase pump. J. Pet. Sci. Eng. 2018, 169, 471-484.

38. Liu, M.; Tan, L.; Cao, S.L. Design Method of Controllable Blade Angle and Orthogonal Optimization of Pressure Rise for a Multiphase Pump. Energies 2018, 11, 1048. [CrossRef]

39. Zhang, J.S.; Tan, L. Energy Performance and Pressure Fluctuation of a Multiphase Pump with Different Gas Volume Fractions. Energies 2018, 11, 1216. [CrossRef]

40. Hu, K.; Li, Z.B. Detailed Explanation of ANSYS ICEM CFD Engineering Example; The People's Posts and Telecommunications Press: Beijing, China, 2014; pp. 207-212, 313-319.

41. Liu, X.R. Research on the Characteristics in Flow Path Tapered Axial-flow Multiphase Pump; Lanzhou University of Technology: Lanzhou, China, 2019.

42. Zhang, T.; Zhu, X.J.; Peng, F.; Min, S.S. Analysis of effect of near-wall treatments on numerical computation of turbulent flow. J. Nav. Univ. Eng. 2013, 25, 108-112.

43. Menter, F.R. Two-Equation Eddy-Viscosity turbulence models for engineering applications. AIAA J. 1994, 32, 1598-1605. [CrossRef]

44. Guan, X.F. Modern Pumps Theory and Design; China Aerospace Publishing House: Beijing, China, 2011; 845p.

45. Ma, X.J.; Zhao, J.; Shao, L. Influence of airfoil selection on performance of oil-gas multiphase pump. Oil Gas. Storage Transp. 2009, 37, 17-19.

46. Kim, J.H.; Lee, H.C. Improvement of Hydrodynamic Performance of a Multiphase Pump Using Design of Experiment Techniques. J. Fluids Eng. 2015, 137, 081301. [CrossRef]

47. Wang, F.J. Computational Fluid Dynamics Analysis: Principles and Applications of CFD Software; Tsinghua University Press: Beijing, China, 2004; pp. 24-29. 